\title{
ON THE SOLUTION OF THE PROBLEM OF SCATTERING OF SURFACE WATER WAVES BY A SHARP DISCONTINUITY IN THE SURFACE BOUNDARY CONDITIONS
}

\author{
A. CHAKRABARTI ${ }^{1}$
}

(Received 7 August 1998; revised 13 January 1999)

\begin{abstract}
Closed-form analytical expressions are derived for the reflection and transmission coefficients for the problem of scattering of surface water waves by a sharp discontinuity in the surface-boundary-conditions, for the case of deep water. The method involves the use of the Havelock-type expansion of the velocity potential along with an analysis to solve a Carleman-type singular integral equation over a semi-infinite range. This method of solution is an alternative to the Wiener-Hopf technique used previously.
\end{abstract}

\section{Introduction}

The problem of scattering of surface water waves in the two-dimensional linearised theory has created several challenges to a large group of workers in applied mathematics (see Stoker [14], Newman [10], Ursell [15], etc.) interested in developing analytical methods of handling a class of mixed boundary-value problems associated with Laplace's equation in two dimensions under varieties of mixed boundary conditions. Recently, Evans and Linton [2] examined the problem of determining the reflection and transmission coefficients involving scattering of surface water waves in a finite depth fluid, on whose surface two different impedance-type boundary conditions are prescribed on the two sides of a fixed position. The mathematical complexities associated with such mixed boundary-value problems for elliptic-type partial differential equations have been known for a long time (see Weitz and Keller [17], Keller and Weitz [7]).

In the present paper, we have described a method involving the Havelock-type expansion (see Ursell [15]) of the velocity potential, for the case of water of infinite

\footnotetext{
${ }^{1}$ Department of Mathematics, Indian Institute of Science, Bangalore 560 012, India; e-mail: alok@math.iisc.ernet.in

(C) Australian Mathematical Society 2000, Serial-fee code 0334-2700/00
} 
depth, on whose surface there exists a single point of discontinuity so that, on the two sides of this point, two different impedance-type boundary conditions are to be met. In order to visualize such boundary conditions, one may imagine that one half of the surface is free to the atmosphere and the other half is covered by an inertial surface of constant area density (see Gabov et al. [4]). We can also imagine, for the sake of realizing the boundary conditions used here, that the surface of the fluid is composed of two different types of distribution of floating ice particles (see Weitz and Keller [17]) on either side of a fixed position on it and it may be remarked that, as pointed out by Evans [1], though there are limitations on the use of such a model it provides a useful check of limiting cases for more general boundary-value problems involving Laplace's equation. Different models involving ice-covered surfaces have been proposed in an investigation by Fox and Squire [3] (see the other references cited in [3] also).

The particular mixed boundary-value problem considered here also arises in the study of propagation of interface waves (see Gabov et al. [4]) at the interface of two immiscible fluids of infinite extent, where one half of the interface is covered by an inertial surface of known constant area density and the other half is a free separation boundary of the two fluids. Thus, the importance of the particular model chosen in the present study is that the same boundary-value problem arises in many other situations.

The problem under consideration is reduced, in the present paper, to that of solving a Carleman-type singular integral equation over a semi-infinite range, whose solution is determined by utilizing the techniques available in the books of Muskhelishvili [9] and Gakhov [5]. The full solution is finally determined in a neat analytical form and the closed-form expressions for the reflection and transmission coefficients are also presented. A solution has been obtained before, by Peters [11], using the Wiener-Hopf technique. Whilst it is well-known that the Wiener-Hopf technique is undoubtedly an extremely powerful method, capable of handling more general boundary value problems than the one considered here, we here demonstrate the use of Fourier analysis and Carleman-type singular integral equations, even though the analysis is rather long-winded. The present work deals with the simplest case of normally incident waves. The case of obliquely incident waves is yet to be examined by the method described here.

As has already been mentioned, the problem of the present work involves fluid of infinite depth as compared to the work of Evans and Linton [2], where the problem for fluid of finite depth has been solved using the residue calculus method of Mitra and Lee [8] to determine the reflection and transmission coefficients. The analysis of Evans and Linton [2] can also be utilized, with care, for the problem of the present paper. 


\section{The statement of the problem and reduction to an integral equation}

We shall assume that the flow of the fluid under our consideration is two-dimensional, irrotational, inviscid, incompressible and time-harmonic (with time-dependence $e^{-i \omega t}, i^{2}=-1, \omega>0$ ) in nature. Then, using Cartesian $x, y$ coordinates, with the $x$-axis in the horizontal direction and the positive $y$-axis in the downward vertical direction respectively, so that the fluid occupies the semi-infinite region $y>0$, the mathematical problem is that of the determination of the velocity potential $\Phi(x, y, t)=\operatorname{Re}\left\{\phi(x, y) e^{-i \omega t}\right\}$, in which $\phi$ satisfies the following equation and conditions:

(i) the p.d.e. $\frac{\partial^{2} \phi}{\partial x^{2}}+\frac{\partial^{2} \phi}{\partial y^{2}}=0$, in $y>0$;

(ii) the impedance-type boundary conditions on the surface of deep water of constant density

$$
\begin{aligned}
& \text { (a) } \frac{\partial \phi}{\partial y}+K_{1} \phi=0, \\
& \text { (b) } \frac{\partial \phi}{\partial y}+K_{2} \phi=0,
\end{aligned}
$$

where $\alpha$ represents a fixed real number, with $K_{1}(>0)$ and $K_{2}(>0)$ representing the wave numbers corresponding to surface waves on the two sides $x<\alpha$ and $x>\alpha$, respectively;

(iii) $\phi, \operatorname{grad} \phi \rightarrow 0$, as $y \rightarrow \infty$;

(iv) $\phi \rightarrow e^{i K_{1} x-K_{1} y}+R e^{-i K_{1} x-K_{1} y}$, as $x \rightarrow-\infty$; and

(v) $\phi \rightarrow T e^{i K_{2} x-K_{2} y}$, as $x \rightarrow+\infty$,

where $R$ and $T$ denote the complex "reflection" and "transmission" coefficients of the scattering problem under consideration.

The method of solution of the above mixed boundary-value problem is as follows. We write $\phi=\phi_{1}$ for the region $(x<\alpha, y>0)$ and $\phi=\phi_{2}$ for the region $(x>\alpha$, $y>0$ ), respectively, and set, using Havelock-type expansions (see Ursell [15]),

$$
\begin{aligned}
\phi_{1}= & e^{-K_{1} y+i K_{1} x}+R e^{-K_{1} y-i K_{1} x} \\
& +\frac{2}{\pi} \int_{0}^{\infty} \frac{A(\xi)}{\left(\xi^{2}+K_{1}^{2}\right)} e^{\xi(x-\alpha)}\left(\xi \cos \xi y-K_{1} \sin \xi y\right) d \xi, \quad \text { for } x<\alpha, y>0
\end{aligned}
$$

and

$$
\begin{aligned}
\phi_{2}= & T e^{-K_{2} y+i K_{2} x} \\
& +\frac{2}{\pi} \int_{0}^{\infty} \frac{B(\xi)}{\left(\xi^{2}+K_{2}^{2}\right)} e^{-\xi(x-\alpha)}\left(\xi \cos \xi y-K_{2} \sin \xi y\right) d \xi, \quad \text { for } x>\alpha, y>0,
\end{aligned}
$$


where $A(\xi)$ and $B(\xi)$ represent two unknown functions to be determined along with the other two unknown complex constants $R$ and $T$.

The conditions of continuity of $\phi$ and $\partial \phi / \partial x$ across the line $x=\alpha$ give that

$$
\begin{gathered}
e^{-K_{1} y+i K_{1} \alpha}+R e^{-K_{1} y-i K_{1} \alpha}+\frac{2}{\pi} \int_{0}^{\infty} \frac{A(\xi)}{\left(\xi^{2}+K_{1}^{2}\right)}\left(\xi \cos \xi y-K_{1} \sin \xi y\right) d \xi \\
=T e^{-K_{2} y+i K_{2} \alpha}+\frac{2}{\pi} \int_{0}^{\infty} \frac{B(\xi)}{\left(\xi^{2}+K_{2}^{2}\right)}\left(\xi \cos \xi y-K_{2} \sin \xi y\right) d \xi
\end{gathered}
$$

and

$$
\begin{gathered}
i K_{1}\left[e^{-K_{1} y+i K_{1} \alpha}-R e^{-K_{1} y-i K_{1} \alpha}\right]+\frac{2}{\pi} \int_{0}^{\infty} \frac{\xi A(\xi)}{\left(\xi^{2}+K_{1}^{2}\right)}\left(\xi \cos \xi y-K_{1} \sin \xi y\right) d \xi \\
=i K_{2} T e^{-K_{2} y+i K_{2} \alpha}-\frac{2}{\pi} \int_{0}^{\infty} \frac{\xi B(\xi)}{\left(\xi^{2}+K_{2}^{2}\right)}\left(\xi \cos \xi y-K_{2} \sin \xi y\right) d \xi
\end{gathered}
$$

The improper integrals appearing in the relations (2.1) and (2.2) and all such integrals appearing in the rest of the analysis will be understood as the limits of appropriately designed convergent integrals (see (2.6)).

We next use the following "Mixed Fourier Transform Pair" (see Sneddon [12]), also known as Havelock's expansion theorem (see Ursell [15]):

"If

$$
\int_{0}^{\infty} f(t)(\xi \cos \xi t-K \sin \xi t) d t=F(\xi), \quad \xi>0, K>0
$$

with

$$
f(t)=\frac{2}{\pi} \int_{0}^{\infty} \frac{F(\xi)}{\xi^{2}+K^{2}}(\xi \cos \xi t-K \sin \xi t) d \xi+C_{0} e^{-K t}, \quad t>0
$$

where $f(t)$ and its derivative are continuous and integrable in the range $(0, \infty)$."

Then, using (2.3), we determine, from (2.1) and (2.2), that

$$
\begin{aligned}
A(\xi)= & T e^{i K_{2} \alpha}\left[\frac{\left(K_{2}-K_{1}\right) \xi}{\xi^{2}+K_{2}^{2}}\right]+\left(\frac{\xi^{2}+K_{1} K_{2}}{\xi^{2}+K_{2}^{2}}\right) B(\xi) \\
& -\frac{2}{\pi}\left(K_{2}-K_{1}\right) \xi \int_{0}^{\infty} \frac{u B(u) d u}{\left(u^{2}+K_{2}^{2}\right)\left(u^{2}-\xi^{2}\right)}
\end{aligned}
$$

and

$$
\begin{aligned}
\xi A(\xi)= & i K_{2} T e^{i K_{2} \alpha}\left[\frac{\left(K_{2}-K_{1}\right) \xi}{\xi^{2}+K_{2}^{2}}\right]-\left(\frac{\xi^{2}+K_{1} K_{2}}{\xi^{2}+K_{2}^{2}}\right) \xi B(\xi) \\
& +\frac{2}{\pi}\left(K_{2}-K_{1}\right) \xi \int_{0}^{\infty} \frac{u^{2} B(u) d u}{\left(u^{2}+K_{2}^{2}\right)\left(u^{2}-\xi^{2}\right)}
\end{aligned}
$$


after interchanging the orders of integration and employing the "generalized" identities:

$$
\begin{aligned}
& \lim _{\epsilon \rightarrow 0} \int_{0}^{\infty} e^{-\epsilon y} \cos u y \cos \xi y d y=\frac{\pi}{2}[\delta(\xi-u)+\delta(\xi+u)] \\
& \lim _{\epsilon \rightarrow 0} \int_{0}^{\infty} e^{-\epsilon y} \sin u y \sin \xi y d y=\frac{\pi}{2}[\delta(\xi-u)-\delta(\xi+u)] \\
& \lim _{\epsilon \rightarrow 0} \int_{0}^{\infty} e^{-\epsilon y} \sin u y \cos \xi y d y=\frac{u}{u^{2}-\xi^{2}}
\end{aligned}
$$

where $\delta(x)$ denotes the well-known Dirac's delta function.

We must keep in mind, in view of the expansion theorem (2.3), that the following relations must also hold good:

$$
\frac{1}{2 K_{1}}\left(e^{i K_{1} \alpha}+R e^{-i K_{1} \alpha}\right)=\frac{T e^{i K_{2} \alpha}}{K_{1}+K_{2}}+\frac{2}{\pi}\left(K_{1}-K_{2}\right) \int_{0}^{\infty} \frac{\xi B(\xi) d \xi}{\left(\xi^{2}+K_{1}^{2}\right)\left(\xi^{2}+K_{2}^{2}\right)}
$$

and

$$
\frac{i}{2}\left(e^{i K_{1} \alpha}-R e^{-i K_{1} \alpha}\right)=\frac{i K_{2} T e^{-i K_{2} \alpha}}{K_{1}+K_{2}}-\frac{2}{\pi}\left(K_{1}-K_{2}\right) \int_{0}^{\infty} \frac{\xi^{2} B(\xi) d \xi}{\left(\xi^{2}+K_{1}^{2}\right)\left(\xi^{2}+K_{2}^{2}\right)} .
$$

The elimination of $A(\xi)$ between (2.4) and (2.5) gives rise to the following equation for the determination of the unknown function $B(\xi)$ :

$$
\begin{gathered}
\left(\frac{\xi^{2}+K_{1} K_{2}}{\xi^{2}+K_{2}^{2}}\right) B(\xi)-\frac{1}{\pi}\left(K_{2}-K_{1}\right) \int_{0}^{\infty} \frac{u B(u) d u}{(u-\xi)\left(u^{2}+K_{2}^{2}\right)} \\
=-\frac{\left(K_{2}-K_{1}\right) T e^{i K_{2} \alpha}}{2\left(\xi+i K_{2}\right)}, \quad \xi>0,
\end{gathered}
$$

in which the singular integral is understood in the sense of its Cauchy principal value (see Gakhov [5]). All singular integrals appearing in the paper are understood similarly.

Reusing (2.9) in (2.4) gives

$$
A(\xi)=\frac{T\left(K_{2}-K_{1}\right) e^{i K_{2} \alpha}}{2\left(\xi-i K_{2}\right)}+\frac{\left(K_{2}-K_{1}\right)}{\pi} \int_{0}^{\infty} \frac{u B(u) d u}{(u+\xi)\left(u^{2}+K_{2}^{2}\right)}, \quad \xi>0 .
$$

Thus, the problem of determination of the two unknowns $A(\xi)$ and $B(\xi)$ has been reduced, basically, to the problem of solving a single Carleman-type singular integral equation over a semi-infinite range, as given by (2.9), which can be cast into the form:

$$
C(\xi) \lambda(\xi)+\frac{1}{\pi} \int_{0}^{\infty} \frac{\lambda(u) d u}{u-\xi}=\frac{T e^{i K_{2} \alpha}}{2\left(\xi+i K_{2}\right)}, \quad \xi>0
$$


where

$$
\lambda(u)=\frac{u B(u)}{\left(u^{2}+K_{2}^{2}\right)}
$$

and

$$
C(u)=\frac{\left(u^{2}+K_{1} K_{2}\right)}{u\left(K_{1}-K_{2}\right)}
$$

We shall next solve the integral equation (2.11) and determine the full solution of the problem under consideration.

\section{The full solution}

The singular integral equation (2.11) can be solved by using the techniques available in the books of Muskhelishvili [9] and Gakhov [5]. We describe here the method of solution rather briefly (see Spence [13] and Varley and Walker [16] also).

Introducing a sectionally analytic function $\Lambda(\xi)$, as defined by the relation

$$
\Lambda(\zeta)=\frac{1}{2 \pi i} \int_{0}^{\infty} \frac{\lambda(u) d u}{u-\zeta}
$$

and utilizing the Plemelj-Sokhotskii formulae as given by

$$
\Lambda^{ \pm}(\xi)= \pm \frac{1}{2} \lambda(\xi)+\frac{1}{2 \pi i} \int_{0}^{\infty} \frac{\lambda(u) d u}{u-\xi}
$$

(2.11) can be expressed in the form

$$
[C(\xi)+i] \Lambda^{+}(\xi)-[C(\xi)-i] \Lambda^{-}(\xi)=\frac{T e^{i K_{2} \alpha}}{2\left(\xi+i K_{2}\right)}, \quad \xi>0
$$

which represents a Riemann-Hilbert problem for the determination of the function $\Lambda(\zeta)$.

The solution of the problem (3.3) can be easily written down in the form

$$
\Lambda(\zeta)=\frac{T e^{i K_{2} \alpha}}{4 \pi i} \Lambda_{0}(\zeta) \int_{0}^{\infty} \frac{d u /(u-\zeta)}{\Lambda_{0}^{+}(u)[C(u)+i]\left(u+i K_{2}\right)}
$$

where $\Lambda_{0}(\zeta)$ represents the solution of the homogeneous problem (3.3) which is analytic and non-zero in the complex $\zeta$-plane cut along the positive real axis and which is such that $\Lambda_{0}(\zeta) \sim 0(1)$ as $|\zeta| \rightarrow \infty$, producing $\Lambda(\zeta) \sim 0(1 /|\zeta|)$ as $|\zeta| \rightarrow \infty$, as is to be expected of the form (3.1) for the function $\Lambda(\zeta)$. 
The solution of the integral equation (2.11) can be determined by reusing formula (3.2), giving

$$
\lambda(\xi)=\Lambda^{+}(\xi)-\Lambda^{-}(\xi), \quad \xi>0
$$

By using (3.4), (3.5), (3.2), (2.12) and (2.13), we finally determine that the unknown function $B(\xi)$ of our problem has the following representation:

$$
\begin{aligned}
B(\xi)= & T\left(K_{1}-K_{2}\right) e^{i K_{2} \alpha}\left[\frac{\left(\xi^{2}+K_{1} K_{2}\right)}{2\left(\xi+i K_{2}\right)\left(\xi^{2}+K_{1}^{2}\right)}\right. \\
& \left.-\frac{\left(K_{1}-K_{2}\right)}{2 \pi}\left(\frac{\xi-i K_{2}}{\xi-i K_{1}}\right) \Lambda_{0}^{+}(\xi) \int_{0}^{\infty} \frac{u d u /(u-\xi)}{\Lambda_{0}^{+}(u)\left(u^{2}+K_{2}^{2}\right)\left(u+i K_{1}\right)}\right]
\end{aligned}
$$

It is clear from this expression, that $B(\xi) \sim 0(1 / \xi)$ as $\xi \rightarrow \infty$, which is a necessity for the validity of the analysis employed in the present work and provides a check at this stage of the solution of the problem at hand.

Also, knowing $B(\xi)$, we can determine the other unknown $A(\xi)$ of the problem, by using (2.10).

Thus, the full solution can be completed, once the two unknown constants $R$ and $T$ are also determined. This task can be carried out successfully as described in the next section, the basis of which lies in the pair of formulae (2.7) and (2.8).

\section{The reflection and transmission coefficients}

Solving (2.7) and (2.8) for the unknowns $R$ and $T$, after utilizing (3.6) for the function $B(\xi)$, we arrive at the following results:

$$
T=\left[e^{i\left(K_{1}-K_{2}\right) \alpha}\right]\left[\frac{1}{1+\frac{2 i}{\pi}\left(K_{2}-K_{1}\right)^{2} \int_{0}^{\infty} \frac{\xi Q(\xi) d \xi}{\left(\xi+i K_{1}\right)\left(\xi^{2}+K_{2}^{2}\right)}}\right]
$$

and

$$
R=\left[e^{2 i K_{1} \alpha}\right]\left[\frac{\left(\frac{K_{1}-K_{2}}{K_{1}+K_{2}}\right)-\frac{2 i}{\pi}\left(K_{2}-K_{1}\right)^{2} \int_{0}^{\infty} \frac{\xi Q(\xi) d \xi}{\left(\xi-i K_{1}\right)\left(\xi^{2}+K_{2}^{2}\right)}}{1+\frac{2 i}{\pi}\left(K_{2}-K_{1}\right)^{2} \int_{0}^{\infty} \frac{\xi Q(\xi) d \xi}{\left(\xi+i K_{1}\right)\left(\xi^{2}+K_{2}^{2}\right)}}\right]
$$

where

$$
\begin{aligned}
Q(\xi)= & \frac{\left(\xi^{2}+K_{1} K_{2}\right)}{2\left(\xi+i K_{2}\right)\left(\xi^{2}+K_{1}^{2}\right)} \\
& -\frac{\left(K_{1}-K_{2}\right)}{2 \pi}\left(\frac{\xi-i K_{2}}{\xi-i K_{1}}\right) \Lambda_{0}^{+}(\xi) \int_{0}^{\infty} \frac{u d u /(u-\xi)}{\Lambda_{0}^{+}(u)\left(u^{2}+K_{2}^{2}\right)\left(u+i K_{1}\right)}
\end{aligned}
$$


with $\Lambda_{0}^{+}(\xi)$ being the 'plus'-limiting value of the function $\Lambda_{0}(\zeta)$, in the usual notation.

Note that (cf. (3.6))

$$
B(\xi)=T\left(K_{1}-K_{2}\right) e^{i K_{2} \alpha} Q(\xi)
$$

It is interesting to observe that all the integrals appearing in the results (4.1), (4.2) and (4.3) can be evaluated in closed form by some clever use of Cauchy's residue theorem, along with the fact that $\Lambda_{0}(\zeta)$ solves the homogeneous problem (3.3), in which $C(\xi)$ is as given by (2.13).

At first we shall evaluate the integral in (4.3) by considering the contour integral

$$
\tilde{I}_{1}=\int_{\Gamma} \frac{d \tau}{\Lambda_{0}(\tau)\left(\tau+i K_{2}\right)(\tau-\zeta)}, \quad \zeta \notin \Gamma,
$$

where $\Gamma$ is a closed contour consisting of a large circular arc along with a loop around the positive real axis. Then, by the aid of the residue theorem as well as the fact that $\Lambda_{0}(\zeta)$ is the solution of the homogeneous problem (3.3), we find that

$$
2 Q(\xi)=\frac{\left(\xi-i K_{2}\right)}{\left(\xi-i K_{1}\right)\left(\xi+i K_{2}\right)}\left[\frac{\Lambda_{0}^{+}(\xi)}{\Lambda_{0}\left(-i K_{2}\right)}\right]
$$

Next, using the result (4.4), the two important integrals

$$
I_{2}=2 \int_{0}^{\infty} \frac{\xi Q(\xi) d \xi}{\left(\xi^{2}+K_{2}^{2}\right)\left(\xi+i K_{1}\right)} \quad \text { and } \quad I_{3}=2 \int_{0}^{\infty} \frac{\xi Q(\xi) d \xi}{\left(\xi^{2}+K_{2}^{2}\right)\left(\xi-i K_{1}\right)}
$$

can be evaluated, by considering two more contour integrals

$$
\tilde{I}_{2}=\int_{\Gamma} \frac{\Lambda_{0}(\tau) d \tau}{(\tau-\zeta)\left(\tau+i K_{1}\right)\left(\tau+i K_{2}\right)}, \quad \zeta \notin \Gamma,
$$

and

$$
\tilde{I}_{3}=\int_{\Gamma} \frac{\Lambda_{0}(\tau) d \tau}{(\tau-\zeta)\left(\tau-i K_{1}\right)\left(\tau+i K_{2}\right)}, \quad \zeta \notin \Gamma,
$$

in which $\Gamma$ is the same closed contour as used in (4.5).

We easily derive that

and

$$
\left.\begin{array}{l}
I_{2}=\frac{\pi}{i\left(K_{1}-K_{2}\right)^{2}}\left[\frac{\Lambda_{0}\left(-i K_{1}\right)}{\Lambda_{0}\left(-i K_{2}\right)}-1\right] \\
I_{3}=\frac{\pi}{i\left(K_{1}^{2}-K_{2}^{2}\right)}\left[1-\frac{\Lambda_{0}\left(i K_{1}\right)}{\Lambda_{0}\left(-i K_{2}\right)}\right] .
\end{array}\right\}
$$


Finally, using (4.6) in (4.1) and (4.2), we find that

$$
T=\left[\frac{\Lambda_{0}\left(-i K_{2}\right)}{\Lambda_{0}\left(-i K_{1}\right)}\right] e^{i\left(K_{1}-K_{2}\right) \alpha} \quad \text { and } \quad R=\left(\frac{K_{1}-K_{2}}{K_{1}+K_{2}}\right)\left[\frac{\Lambda_{0}\left(i K_{1}\right)}{\Lambda_{0}\left(-i K_{1}\right)}\right] e^{2 i K_{1} \alpha} .
$$

It is, perhaps, worth mentioning that, up to this point of our analysis, no explicit expression for the sectionally analytic function $\Lambda_{0}(\zeta)$ is required.

Now, for the particular problem at hand, and for the choice of the two wave numbers $K_{1}$ and $K_{2}$, for which $K_{1}>K_{2}$, we can determine the function $\Lambda_{0}(\zeta)$ by using the relation (see Muskhelishvili [9] and Varley and Walker [16]):

$$
\begin{aligned}
\Lambda_{0}(\zeta)=\exp \left[\frac{1}{2 \pi i}\{\right. & \int_{0}^{\infty} \frac{\left(\log \left(\frac{t-i K_{1}}{t+i K_{1}}\right)-2 \pi i\right) d t}{t-\zeta} \\
& \left.\left.-\int_{0}^{\infty} \frac{\left(\log \left(\frac{t-i K_{2}}{t+i K_{2}}\right)-2 \pi i\right) d t}{t-\zeta}\right\}\right], \quad \zeta \notin(0, \infty)
\end{aligned}
$$

Then, some standard and simple manipulations lead to the interesting results given by

$$
|T|=\left|\frac{\Lambda_{0}\left(-i K_{2}\right)}{\Lambda_{0}\left(-i K_{1}\right)}\right|=\frac{2\left(K_{1} K_{2}\right)^{1 / 2}}{K_{1}+K_{2}} \quad \text { and } \quad|R|=\left|\frac{K_{1}-K_{2}}{K_{1}+K_{2}}\right|,
$$

which satisfy the requirement that

$$
|R|^{2}+|T|^{2}=1
$$

The following two results were needed in the above manipulations (see Gradshteyn and Rhyzik [6]):

$$
\int_{0}^{\pi / 2} \log (\sin \theta) d \theta=-\frac{\pi}{2} \log 2
$$

and

$$
\int_{0}^{\pi / 2} \log \left(K_{1}^{2}+K_{2}^{2} \tan ^{2} \theta\right) d \theta=\pi \log \left(K_{1}+K_{2}\right)
$$

\section{Acknowledgements}

The idea of solving the problem of this paper in the present manner occurred while visiting the Tata Institute of Fundamental Research (TIFR) in Bombay during the 
period 26 June to 17 July 1995. My sincere thanks to Professor S. G. Dani, Dean of the School of Mathematics at TIFR, for his invitation to visit.

I thank the referees for their many valuable comments and suggestions which have improved the presentation of the paper considerably.

\section{References}

[1] D. V. Evans, "The solution of a class of boundary value problems with smoothly varying boundary conditions", Q. J. Mech. Appl. Math. 38 (1994) 521-536.

[2] D. V. Evans and C. M. Linton, "On the step approximation for water wave problems", J. Fluid Mech. 278 (1994) 229-249.

[3] C. Fox and V. A. Squire, "On the oblique reflection and transmission of ocean waves at shore fast sea ice", Philos. Trans. Roy. Soc. London, Ser. A. 347 (1994) 185-218.

[4] S. A. Gabov, A. G. Sveshnikov and A. K. Shatov, "Dispersion of internal waves by an obstacle floating on the boundary separating two liquids", Prikl. Mat. Mech. 53 (1989) 727-730, (Russian).

[5] F. D. Gakhov, Boundary value problems (Pergamon, Oxford, 1966).

[6] I. M. Gradshteyn and I. S. Rhyzik, Tables of integrals, infinite series and products (Academic Press, 1980).

[7] J. B. Keller and M. Weitz, "Reflection and transmission coefficients of water waves entering or leaving an ice-field", Comm. Pure Appl. Math. 6 (1953) 415-417.

[8] R. Mitra and S. W. Lee, Analytical techniques in the theory of guided wave (Mac-Millan, 1971).

[9] N. I. Muskhelishvili, Singular integral equations (Gröningen, Holland, Nordhoff, 1953).

[10] J. N. Newman, "Propagation of water waves over an infinite step", J. Fluid Mech. 23 (1965) $399-415$.

[11] A. S. Peters, "The effect of a floating mat on water waves", Comm. Pure Appl. Math. 3 (1950) 319-354.

[12] I. N. Sneddon, The use of integral transforms (Tata McGraw Hill, New Delhi, 1974).

[13] D. A. Spence, "The lift coefficient of a thin jet flapped wing, II: A solution of the integro-differential equation for the slope of the jet”, Proc. R. Soc., Ser. A 261 (1961) 97-118.

[14] J. J. Stoker, Water waves (Wiley Interscience, 1957).

[15] F. Ursell, "The effect of a fixed vertical barrier on surface water waves in deep water", Proc. Cambridge Philos. Soc. 43 (1947) 374-382.

[16] E. Varley and J. D. A. Walker, "A method for solving singular integro-differential equations", IMA J. of Appl. Math. 43 (1989) 11-45.

[17] M. Weitz and J. B. Keller, "Reflection of water waves from floating ice in water of finite depth", Comm. Pure Appl. Math. 3 (1950) 305-318. 